\title{
Management of a Ruptured Mycotic Superficial Femoral Artery Aneurysm
}

\author{
Nisheeth Kansal ${ }^{a}$, Kemal Kemal ${ }^{\mathrm{a}}$, Sydney Parker ${ }^{\mathrm{a}}$, Reem Salmanª \\ Vish Bhattacharya ${ }^{a, b}$
}

\begin{abstract}
Aneurysms of peripheral arteries of the leg like the iliac, superficial femoral or popliteal arteries are quite rare. They often tend to coexist or develop later in other vessels in patients who have been treated at one site. They usually present with symptoms of compression of adjacent structures usually the veins or nerves or by distal embolization of the vessels causing a trash foot. It is extremely rare for them to present with a rupture. If this rare event does occur, one has to have strong suspicion of a mycotic (infected) aneurysm. We present a rare case of a ruptured superficial artery aneurysm and its successful management.
\end{abstract}

Keywords: Rupture mycotic; Aneurysm; Superficial femoral artery

\section{Introduction}

Peripheral mycotic aneurysms are extremely rare and present unique management challenges. The evolution of a mycotic aneurysm is multifactorial and is usually due to either septic emboli, direct inoculation of bacteria following intimal disruption, migration through perivascular lymphatics, or vasa vasorum or an interaction of all of the above [1].

They are predominantly reported in literature as being located in axial arteries predominantly in the aorta and rarely in the peripheral vessels [2].

Several treatment modalities have been described for management of these mycotic aneurysms including open surgical approach [3], open approach with tourniquet application and limb exsanguination [4] and endovascular approach [5].

We present the unique challenges presented to us in this case. Our case was unique in that this patient had previously

Manuscript accepted for publication January 08, 2016

aDepartment of Vascular and General Surgery, Queen Elizabeth Hospital, Gateshead, UK

bCorresponding Author: Vish Bhattacharya, Queen Elizabeth Hospital, Gateshead, NE96SX, UK. Email: vbhatta@yahoo.com

doi: http://dx.doi.org/10.14740/jmc2408w had a femoral to distal artery bypass for a popliteal artery aneurysm.

\section{Case Report}

An 80-year-old gentleman presented to the department with a pulsatile mass in his thigh causing pain and swelling of his leg. He had previously had a femoral to distal popliteal artery bypass surgery for a $3 \mathrm{~cm}$ popliteal artery aneurysm that was successfully ligated both proximally and distally during the exclusion bypass procedure carried out 9 months previously.

The bypass had been carried out using a composite graft, which was made of Dacron proximally, and a vein cuff distally. His long saphenous vein had been noted to be unsuitable for a total vein graft and hence the use of a composite graft.

On examination in the emergency department, he was noted to have a $7 \times 8 \mathrm{~cm}$ pulsatile mass over his distal medial thigh that was warm and tender. His right foot was warm and had a palpable dorsalis pedis pulse with no evidence of ischemia (Fig. 1).

Blood tests on admission revealed a white cell count of $11.5 \times 10^{9} / \mathrm{L}$ and a C-reactive protein of $246 \mathrm{mmol} / \mathrm{L}$. He underwent an urgent septic screen and was started on a prophylactic dose of low molecular weight heparin, intravenous teicoplanin, linezolid and aztreonam.

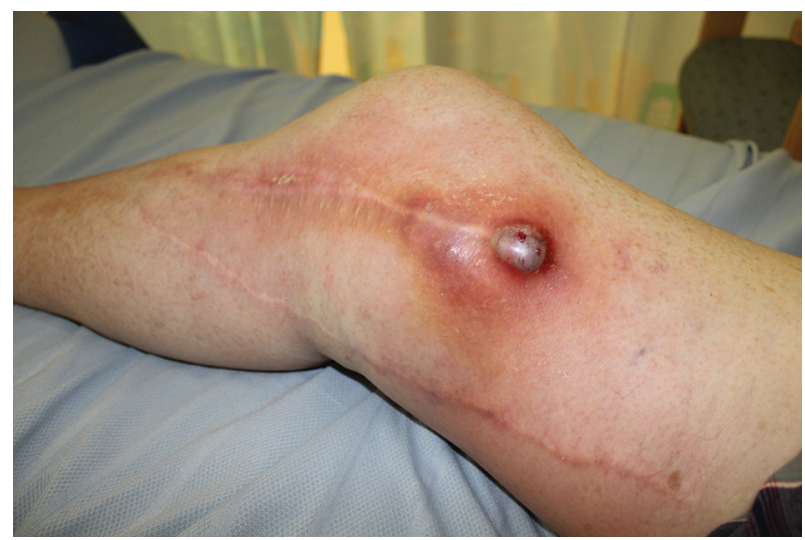

Figure 1. Ruptured superficial femoral artery aneurysm eroding through the skin. 


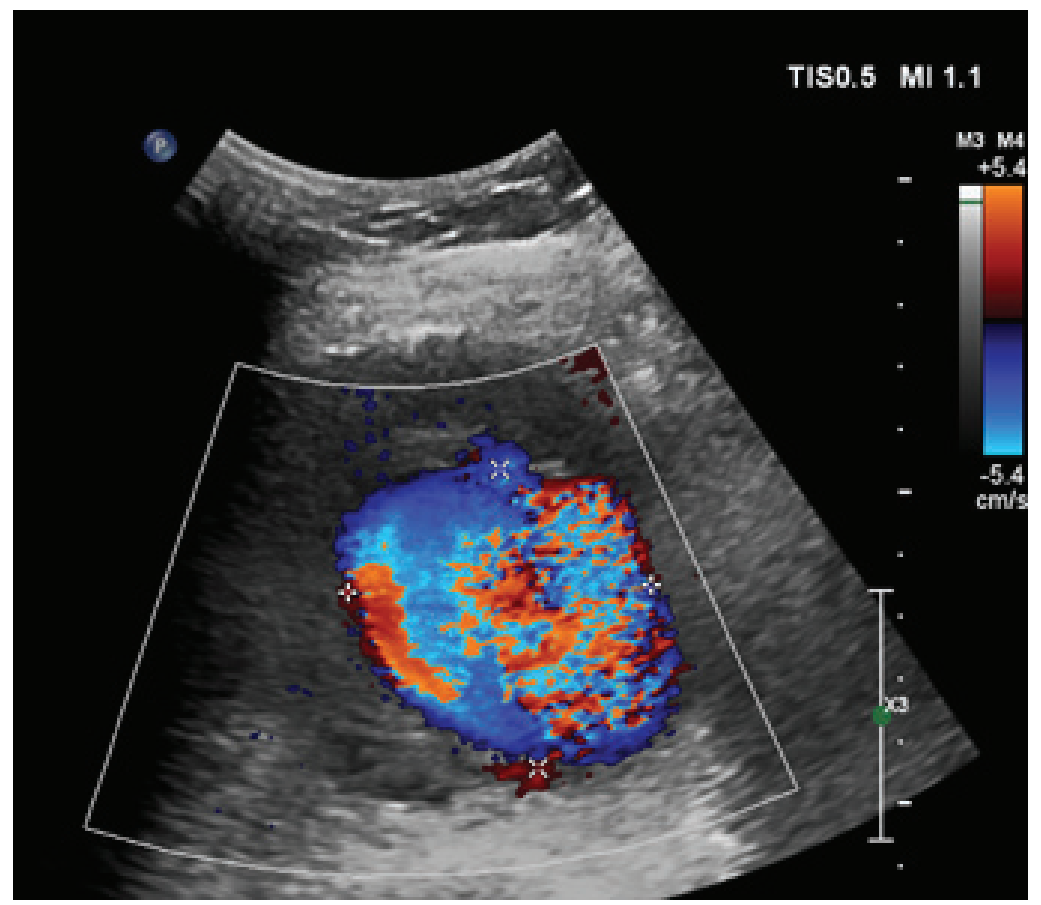

Figure 2. Duplex scan of superficial femoral artery aneurysm showing turbulent flow and thrombus.

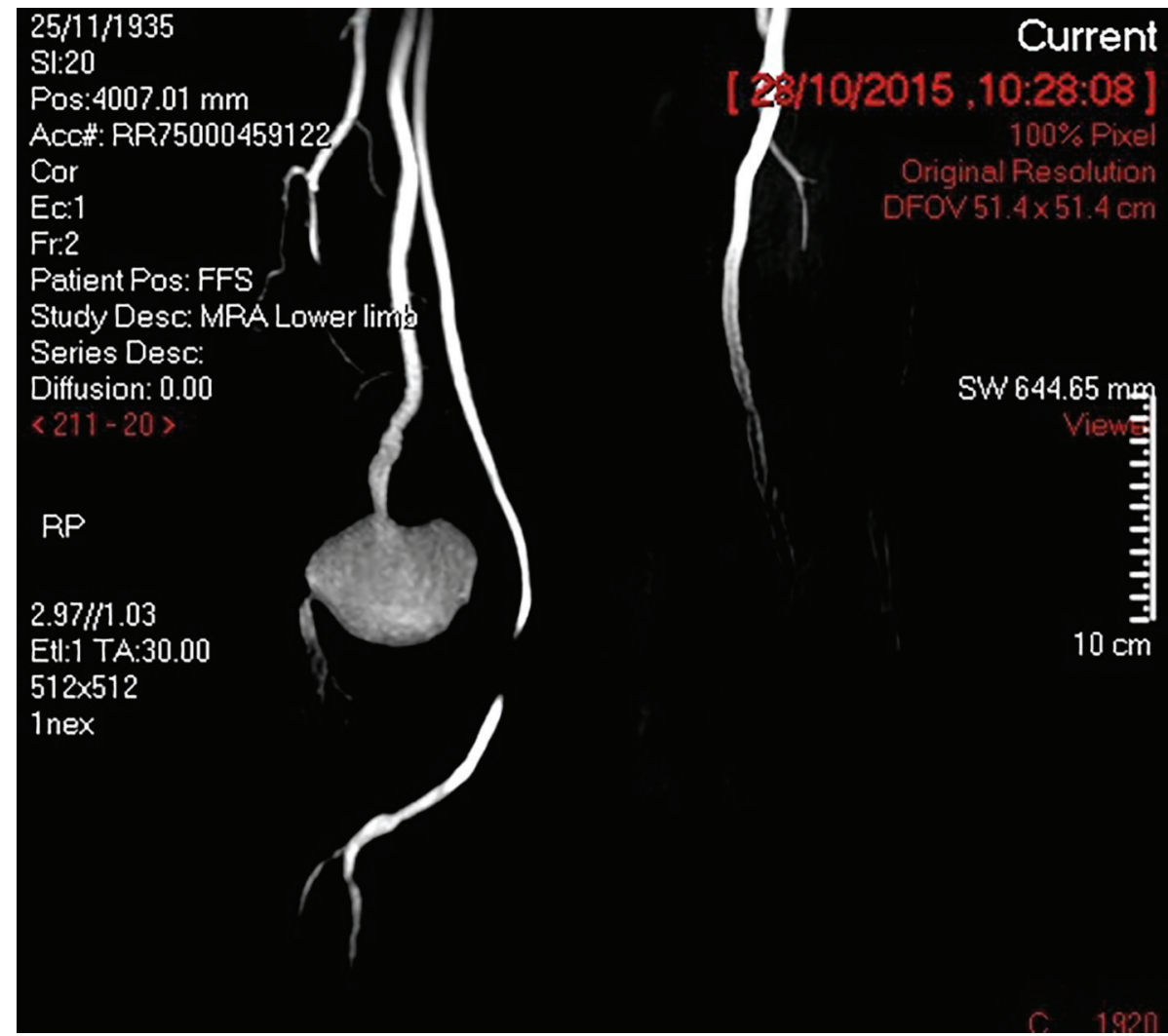

Figure 3. Magnetic resonance angiogram showing large SFA aneurysm and intact previous femoral to distal popliteal graft. 


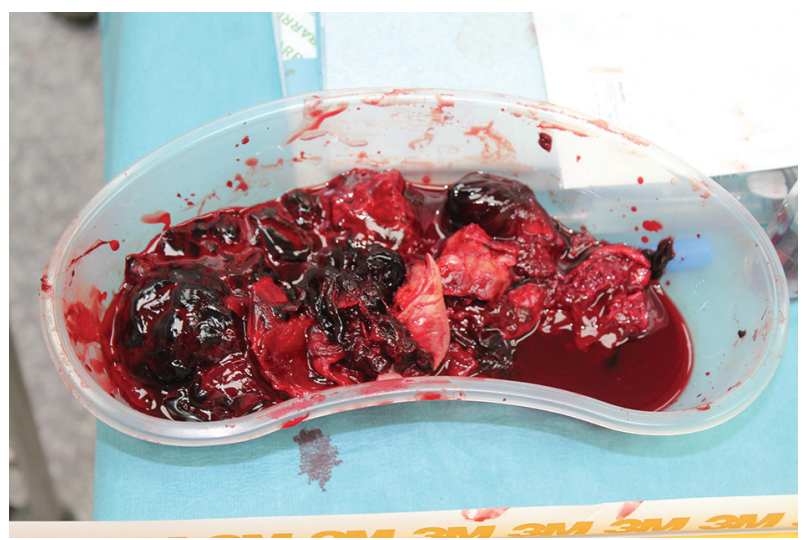

Figure 4. Thrombus and clots removed at surgery.

\section{Investigations}

It was initially suspected that he might have had a pseuodoaneurysm of the graft itself. The duplex scan however revealed that the pulsatile mass was a $7 \mathrm{~cm}$ aneurysm of the distal superficial artery proximal to where the popliteal aneurysm had been ligated previously (Fig. 2). The old femoral popliteal graft was patent with no evidence of a pseudoaneurysm.

A magnetic resonance angiogram confirmed these findings. It showed that the femoral artery aneurysm had a large amount of thrombus and that there was no outflow tract. The previous femoral popliteal graft was found to be completely normal in diameter and patency and was separate from this aneurysm and in a more medial tissue plane (Fig. 3).

\section{Management}

He was operated as an emergency as his pain and swelling had worsened overnight and the aneurysm appeared to be eroding through the skin.

An incision was made over medial aspect of the thigh. There was a large amount of clots noted around the ruptured femoral artery aneurysm. The rupture was however contained within the muscle compartments surrounding the aneurysm.

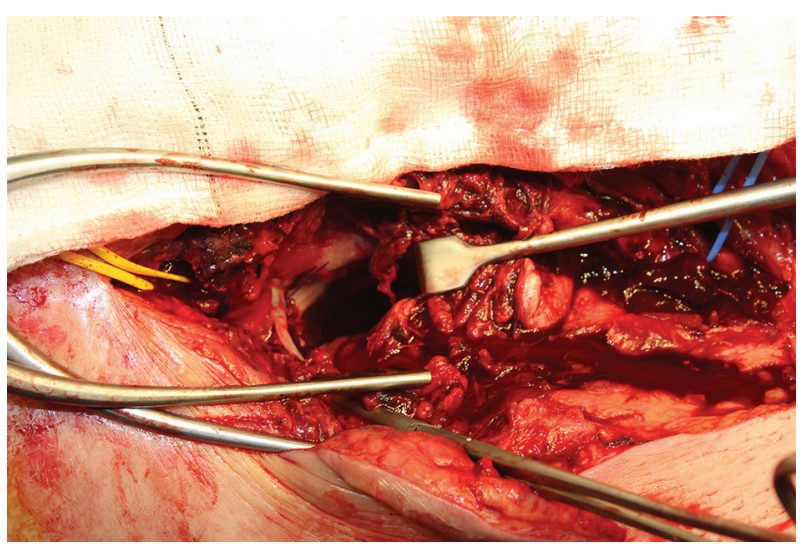

Figure 5. Sac of aneurysm opened and thrombus removed.

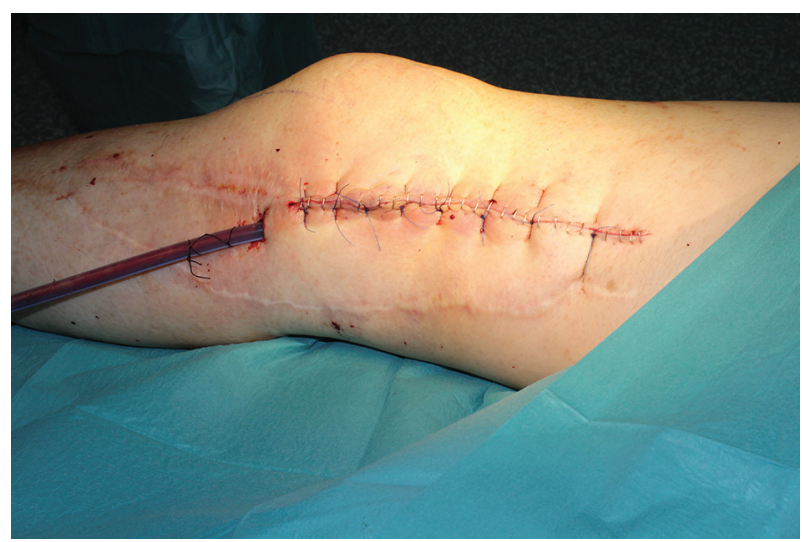

Figure 6. Postoperative image showing resolution of swelling and complete skin closure.

The proximal superficial femoral artery was dissected and ligated with non-absorbable suture. The sac was opened and a large amount of thrombus and clots (approximately 1,000 mL) was removed from the aneurysm sac and sent for microbiology (Fig. 4, 5).

The outflow vessels appeared to be two small genicular branches that were transfixed with non-absorbable sutures. Collatemp (lyophilized collagen implant impregnated with the aminoglycoside antibiotic gentamicin) was placed within the remnant of the sac. Fibrillar ${ }^{\mathrm{TM}}$ (absorbable hemostat) was placed inside the wound to further aid hemostasis. One silastic drain was left in situ (Fig. 6).

\section{Outcome and follow-up}

He was returned to the surgical ward postoperatively where he made a good recovery. His inflammatory markers gradually improved over several days. The microbiology culture of the thrombus revealed coagulase negative gram-positive cocci Staphylococcus lugdunensis that is normally a skin commensal. It has been known to be associated with a variety of infections including endocarditis and cerebral aneurysms [6].

He was continued on antibiotic therapy for a further 6 weeks to fully eradicate the organism. He was eventually discharged 12 days postoperatively. At this stage, he was afebrile and the swelling had disappeared, the leg was warm and perfused and he was mobilizing very well.

\section{Discussion}

Management of ruptured peripheral mycotic aneurysms presents a unique set of challenges and carries considerable risk of morbidity and mortality. The presence of local and systemic sepsis further enhances the physiological stress to the patient.

It is well recognized that patients with popliteal artery aneurysms develop aneurysmal disease in other vessels including the aorta, femoral arteries and iliac arteries. These are treated by open or endovascular repair when they reach a suitable size.

Our case is unique in that the superficial femoral artery 
had appeared completely normal in his previous imaging and then had increased in size quite rapidly to $7 \mathrm{~cm}$ and eventually presented as an infected ruptured aneurysm. This suggests that there had been a focus of infection, which could have led it to grow so rapidly to a large size and eventually rupture. He had not undergone any procedure following his previous surgery and therefore the source of the infection remained unknown.

Shakhnovich et al described an open approach included using extremity exsanguination and tourniquet to control hemorrhage during aneurysm ligation, followed by successful arterial reconstruction for a ruptured mycotic infrapopliteal aneurysm [4]. We avoided using a tourniquet, as this would have endangered his previous graft.

The literature suggests that even mycotic aneurysm can be now repaired covered stents [5, 7, 8], coiling [9] and embolization [10] with no apparent risk of inserting foreign material in an infected field. We did not consider an endovascular approach as there was a theoretical risk of infection of a stent. Moreover, there was no distal vessel to catheterize as the aneurysm appeared to be an end artery with no major outflow tract.

Thus an open surgical approach was used involving ligation of the aneurysm and preservation of the previously performed femoral to distal popliteal bypass graft. The patient was last seen mobilizing very well with no evidence of leg swelling and excellent ankle pulses.

\section{Conclusions}

Rapid expansion and rupture of an aneurysm of the femoral artery is rare and needs to be treated urgently with ligation and bypass. Our case report demonstrated that when a bypass is already present, simple ligation and evacuation of the aneurysm is sufficient. Rapid expansion also suggests an infection and investigations should include exclusion of sources of infection, e.g. urinary tract infection, chest infection or endocarditis. Despite extensive investigations, we found that the only possible source of the organism was a skin commensal that could have entered the blood stream and caused the mycotic aneurysm.

\section{References}

1. Jebara VA, Nasnas R, Achouh PE, Tabet G, Kassab R, Karam B, Rassi I. Mycotic aneurysm of the popliteal artery secondary to tuberculosis. A case report and review of the literature. Tex Heart Inst J. 1998;25(2):136-139.

2. Barros MV, Penna JT, Henriques JF, Roquette-Reis F, Labropoulos N. Infrapopliteal mycotic aneurysm caused by endocarditis--case report and literature review. Echocardiography. 2010;27(1):77-79.

3. Ferrero E, Ferri M, Viazzo A, Gaggiano A, Berardi G, Piazza S, Cumbo P, et al. Rupture of a true giant aneurysm of the posterior tibial artery: a huge size of $6 \mathrm{~cm}$ on diameter. Ann Vasc Surg. 2010;24(8):1134 e1139-1113.

4. Shakhnovich I, Seabrook GR, Brown KR, Lewis BD, Rossi PJ. Ruptured mycotic infrapopliteal aneurysm. J Vasc Surg. 2013;58(1):205-207.

5. Bani-Hani MG, Elnahas L, Plant GR, Ward A, Moawad M. Endovascular management of ruptured infected popliteal artery aneurysm. J Vasc Surg. 2012;55(2):532-534.

6. Tamdy A, El Louali F, Ounzar M, Fettouhi H, Hajkacem H, Fellat I, Zarzur J, et al. Multiple mycotic aneurysms reveal Staphylococcus lugdunensis endocarditis in a young patient. Heart Lung. 2011;40(4):352-357.

7. Bell RE, Taylor PR, Aukett M, Evans GH, Reidy JF. Successful endoluminal repair of an infected thoracic pseudoaneurysm caused by methicillin-resistant Staphylococcus aureus. J Endovasc Ther. 2003;10(1):29-32.

8. Rojas A, Mertens R, Arbulo D, Garcia P, Labarca J. Multiple mycotic aneurysms due to penicillin nonsusceptible Streptococcus pneumoniae solved with endovascular repair. Ann Vasc Surg. 2010;24(6):827 e825-828.

9. Barbano B, Gigante A, Zaccaria A, Polidori L, Martina P, Schioppa A, Ferrazza A, et al. True posterior tibial artery aneurysm in a young patient: surgical or endovascular treatment? BMJ Case Rep. 2009;2009

10. Fujimura N, Obara H, Matsumoto K, Kitagawa Y. Mycotic aneurysm of the superior gluteal artery in a patient with bacterial endocarditis: case report and review of the literature. Vascular. 2011;19(1):47-50. 